\title{
Adjuvante Therapie des Kolonkarzinoms
}

\author{
Anke Reinacher-Schick ${ }^{\mathrm{a}}$ Dirk Arnold ${ }^{\mathrm{b}}$ Tanja Trarbach $^{\mathrm{c}} \quad$ Karsten Ridwelski $^{\mathrm{d}}$ \\ Hans-Peter Bruch ${ }^{\mathrm{e}}$ Thomas Kirchner ${ }^{f}$ Stefan Kubicka ${ }^{g}$ Hans-Joachim Schmoll ${ }^{b}$ \\ ${ }^{\text {a }}$ Medizinische Universitätsklinik Knappschaftskrankenhaus, Ruhr-Universität Bochum, \\ ${ }^{\mathrm{b}}$ Klinik für Innere Medizin IV, Onkologie/Hämatologie, Universitätsklinikum Halle (Saale), \\ ${ }^{c}$ Innere Klinik (Tumorforschung), Universitätsklinikum Essen, \\ ${ }^{d}$ Klinik für Allgemein- und Viszeralchirurgie, Klinikum Magdeburg, \\ ${ }^{e}$ Klinik für Chirurgie, Universitätsklinikum Schleswig-Holstein, Campus Lübeck, \\ ${ }^{f}$ Pathologisches Institut der LMU München, \\ ${ }^{g}$ Abteilung Gastroenterologie, Hepatologie und Endokrinologie, Medizinische Hochschule Hannover, Deutschland
}

\section{Schlüsselwörter}

Kolonkarzinom - Adjuvante Therapie .

Mikrosatelliten-Instabilität (MSI) · Ältere Patienten .

Capecitabin · Oxaliplatin

\section{Zusammenfassung}

Das Ziel, die adjuvante Therapie zu verbessern, kann zum einen durch die Entwicklung wirksamerer Substanzen und Schemata erreicht werden, zum anderen durch die Auswahl der geeigneten Patienten nach klinischen und molekularen Faktoren. Im UICC (Union internationale contre le cancer)Stadium II ist die Mikrosatelliten-Instabilität (MSI) ein starker prognostischer Faktor. Ob es sich darüber hinaus auch um einen prädiktiven Marker handelt, wird aufgrund widersprüchlicher Daten derzeit kontrovers diskutiert. Die Frage, ob vor der Therapieentscheidung bei Stadium-II-Patienten der MSI-Status überprüft werden soll, kann deshalb zurzeit nicht einheitlich beantwortet werden. Für die adjuvante Therapie im Stadium III steht mit Capecitabin/Oxaliplatin (XELOX) jetzt ein weiteres, auf dem oralen Prodrug Capecitabin basierendes Protokoll zur Verfügung. Zu der Frage, inwieweit auch ältere Patienten in diesem Stadium von einer Kombinationschemotherapie profitieren, liegen neue - widersprüchliche - Daten vor: Zum einen haben vorläufige Ergebnisse von zwei neuen Studien zu Sicherheitsbedenken geführt, zum anderen wies eine Analyse der "ACCENT Collaborative Group» auf eine geringere Effektivität von «neueren" adjuvanten Protokollen bei Älteren hin. Diese Daten werden durch eine aktuelle Subgruppenanalyse der XELOXA-Studie allerdings relativiert. Die Expertenrunde empfahl deshalb, die Entscheidung, ob man einen über 70-jährigen Patienten nur mit einem (oralen) Fluoropyrimidin oder in Kombination mit Oxaliplatin behandelt, anhand klinischer Parameter wie dem biologischen Alter und den Komorbiditäten des Patienten festzumachen.

\section{Key Words}

Colon cancer - Adjuvant therapy .

Microsatellite instability (MSI) - Older patients .

Capecitabine $\cdot$ Oxaliplatin

\section{Summary}

Adjuvant Therapy in Colon Cancer

The goal of improving adjuvant treatment can be reached in two ways: firstly, by developing more effective drugs and protocols and, secondly, by selecting suitable patients on the basis of clinical and molecular factors. In UICC (Union internationale contre le cancer) stage II, microsatellite instability (MSI) is a strong prognostic factor. Whether it can also be used as a predictive marker is currently a matter of controversy because the available data are contradictory. The question whether or not the MSI status should be checked before treatment decisions are made in stage II patients can therefore not be clearly answered at present. For adjuvant treatment in stage III, with capecitabine/oxaliplatin (XELOX) there is now a new protocol available that is based on the orally administered prodrug capecitabine. With regard to the question of how much older patients in this stage may also benefit from a combination chemotherapy, new - and contradictory - data have emerged recently: firstly, preliminary results of two new studies have given rise to safety concerns and, secondly, an analysis by the 'ACCENT Collaborative Group' indicated lower efficacy of the 'newer' adjuvant protocols in older people. These findings, however, have now been called into question as a result of a new subgroup analysis from the XELOXA study. The expert group therefore recommended that the decision whether to treat patients older than 70 years with an (oral) fluoropyrimidine alone or in combination with oxaliplatin should be based on clinical parameters such as biological age and comorbidities.

\begin{tabular}{ll}
\hline KARGER & ( ) 2010 S. Karger GmbH, Freiburg \\
Fax +497614520714 & Accessible online at: \\
$\begin{array}{l}\text { Information@Karger.de } \\
\text { www.karger.com }\end{array}$ & www.karger.com/onk
\end{tabular}


Kolonkarzinom - molekulare Prognoseparameter und Prädiktoren

Seit dem Meeting der American Society of Clinical Oncology (ASCO) 2009 werden in Bezug auf molekulare Prognoseparameter und Prädiktoren bei Patienten mit Kolonkarzinom vor allem 2 Fragen diskutiert:

- Ist ein Kolonkarzinom im Stadium II molekularbiologisch dasselbe wie ein Kolonkarzinom im Stadium III? Wenn nein, ist der Unterschied klinisch relevant?

- Gibt es prädiktive Marker oder Gensignaturen, aus denen sich therapeutische Konsequenzen ableiten lassen?

Die Identifizierung von prädiktiven Markern für die adjuvante Therapie vor allem im Stadium II eines Kolonkarzinoms ist klinisch relevant. Der Einsatz solcher Marker soll Nebenwirkungen vermeiden und Ressourcen sparen.

Bisher ist aus klinischen Studien bekannt, dass Patienten mit einem Kolonkarzinom im Stadium II in etwa $80 \%$ der Fälle durch die Operation geheilt werden (abhängig vom Vorhandensein bestimmter Risikofaktoren). Etwas mehr als $20 \%$ der Patienten werden, meist innerhalb der ersten 2 Jahre, rezidivieren - vor allem in Form von Fernmetastasen - und konsekutiv an der Erkrankung versterben. Eine systemische Therapie zur Senkung des Rezidivrisikos wäre bei dieser Subgruppe sinnvoll, wobei derzeit noch keine molekularen Marker existieren, die diese Subgruppe selektionieren. Etabliert sind bislang nur bestimmte klinische und histopathologische Risikofaktoren, die helfen sollen, Patienten mit einer besonders ungünstigen Prognose im Stadium II zu identifizieren (Stadium II mit Risikofaktoren: T4, zu wenig untersuchte Lymphknoten, Notfalloperation (Ileus, Perforation) [1]). Diese Patienten sollten adjuvant chemotherapeutisch behandelt werden (Verbesserung etwa 7\%). Werden Stadium-IIPatienten ohne Risikofaktoren postoperativ chemotherapeutisch behandelt, führt dies zu einer Überlebensverbesserung von 3-5\% (Abb. 1, oben). Diese Zahlen gehen auf die QUASAR-Studie [2] zurück und sind statistisch signifikant.

An der britischen QUASAR-Studie hatten 3239 Patienten teilgenommen, davon 91\% Lymphknoten-negative und somit Stadium-II-Patienten. Sie erhielten randomisiert entweder 6 Zyklen einer 5-Fluorouracil (5-FU)-haltigen adjuvanten Chemotherapie (5-FU mit/ohne Levamisol bzw. Folinsäure (FS)) oder wurden nur nachbeobachtet. Nach einer medianen Beobachtungszeit von 5,5 Jahren waren in der Chemotherapiegruppe 311 Todesfälle aufgetreten - versus 370 Todesfälle in der Beobachtungsgruppe. Dieser Unterschied war mit $\mathrm{p}=$ 0,008 signifikant und entsprach einem relativen Überlebensvorteil von $18 \%$ und einem absoluten Überlebensvorteil von 3,6\% durch die adjuvante, 5-FU-basierte Therapie.

Hieraus leiten sich die Empfehlungen der aktuellen S3-Leitlinie [1] ab, wonach «bei Patienten mit einem Kolonkarzinom im Stadium II ohne Risikofaktoren eine adjuvante Chemotherapie durchgeführt werden kann». Da für den Einsatz von Oxaliplatin im «low-risk» Stadium II keine überzeugenden Daten

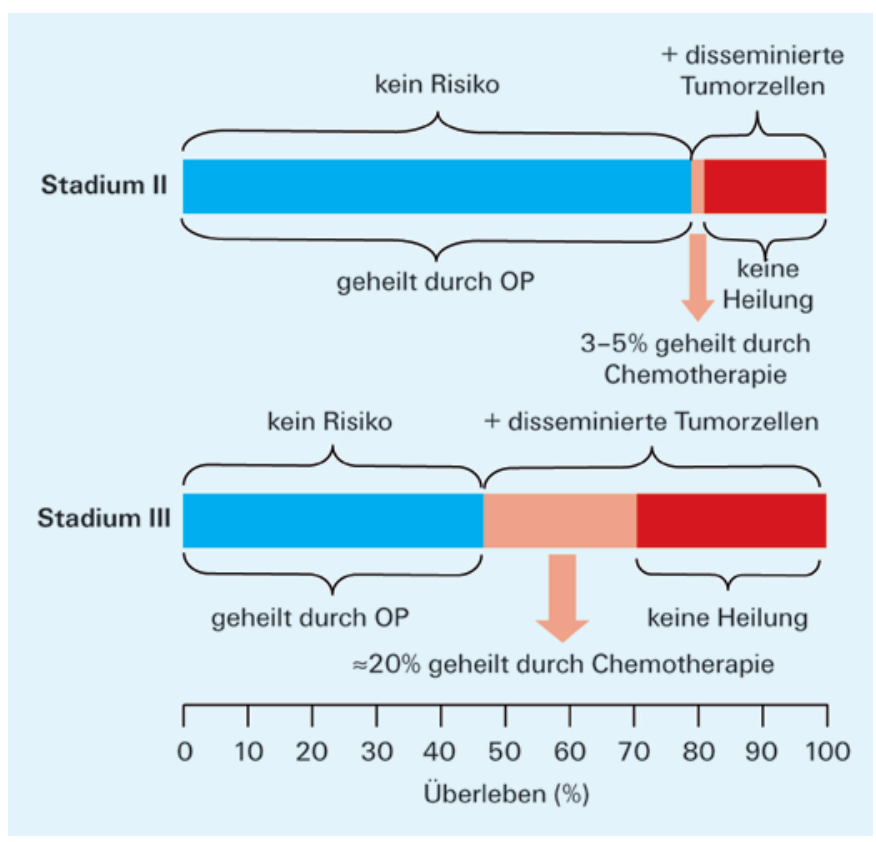

Abb. 1. Kolonkarzinom: Nutzen einer adjuvanten Therapie im Stadium II versus Stadium III (nach Reinacher-Schick et al. [31]).

vorliegen [3, 4], werden hier Fluoropyrimidine als Monotherapie (bevorzugt orale Fluoropyrimidine) empfohlen [1].

Im Stadium III ist die Situation eine andere. Hier kann nur etwa die Hälfte der Patienten durch die Operation geheilt werden. Weitere $20 \%$ des Gesamtkollektivs werden geheilt, wenn alle Patienten postoperativ eine Chemotherapie erhalten (Abb. 1, unten). In der aktuellen S3-Leitlinie war deshalb festgehalten, dass bei Patienten mit einem R0-resezierten Kolonkarzinom im Stadium III eine adjuvante Chemotherapie ohne Altersbeschränkung indiziert ist (Level-A-Empfehlung) [1].

Hilfreich für beide Stadien wären prognostische Marker, anhand derer sich ablesen lässt, bei welchen Patienten disseminierte Tumorzellen vorliegen (denn nur diese Patienten bräuchten eine Chemotherapie), und prädiktive Marker, die vorab aussagen, wer von welcher Therapie profitieren wird.

Im letzten Jahr wurden zu diesem Themenkomplex zahlreiche neue Daten vorgestellt. So zeigte die Pan-European Trials in Alimentary Tract Cancers (PETACC)-3-Studie, dass für Patienten im Stadium II die Mikrosatelliten-Instabilität (MSI) neben der T-Kategorie der stärkste Prognosemarker ist $[5,6]$. Die MSI resultiert aus einer defekten DNA-«Mismatch»-Reparatur (dMMR), und es war schon früher bekannt, dass Patienten mit Tumoren, die eine hochgradige MSI (MSI-H) aufweisen, eine bessere Prognose haben [7] (unter anderem deshalb, weil bei MSI-H-Tumoren viel seltener Fernmetastasen auftreten sollen). Laut PETACC-3 ist der prognostische Wert von MSI aber vor allem bei Patienten mit einem Kolonkarzinom im Stadium II hoch signifikant. Im Stadium III waren andere Prognosemarker wichtiger, woraus die 
Autoren schlussfolgerten, dass es sich bei Stadium II und III um unterschiedliche Erkrankungen handelt. Dem wurde jedoch von einer anderen Arbeitsgruppe widersprochen, die zwar einerseits zeigen konnte, dass Stadium-II-Patienten selbst bei Auftreten eines Tumorrezidivs signifikant bessere Überlebenschancen haben als Stadium-III-Patienten mit einem Rezidiv [8], die aber andererseits bei einer Überprüfung von 375 darmkrebsassoziierten Genen nur bei 5 Genen einen signifikanten Unterschied bezüglich der Genexpression im Stadium II versus III fand [9]. Das Kolonkarzinom im Stadium II und das Kolonkarzinom im Stadium III scheinen also doch eine sehr ähnliche Tumorbiologie zu besitzen.

In einer anderen, methodisch sehr aufwendigen Arbeit gelang die Identifizierung einer prognostischen Gensignatur für Patienten im Stadium II, nicht aber die Identifizierung einer prädiktiven Gensignatur [10]. Auch mittels Coloprint lassen sich nur Aussagen zur Prognose machen [11]. Das heißt, dass nach wie vor auch mittels Gensignaturen nicht vorausgesagt werden kann, wem welche Therapie nützen wird.

Selbst die im letzten Jahr an dieser Stelle ausgesprochene Empfehlung, Patienten im Stadium II routinemäßig auf MSI zu testen, bevor mit einer Fluoropyrimidin-Monotherapie begonnen wird, kann so nicht mehr aufrechterhalten werden. Im letzten Jahr war man davon ausgegangen, dass dem MSI-Status nicht nur eine prognostische, sondern auch eine prädiktive Bedeutung zukommt. Denn mehrere Studien hatten gezeigt, dass sich bei Stadium-II-Patienten mit MSI-H-Tumoren die 5-FU-Therapie signifikant negativ auf das Gesamtüberleben auswirkt [7, 12-14]. Eine weitere Auswertung der PETACC3-Daten bestätigte MSI als starken prognostischen Faktor, kam aber ansonsten zu dem Ergebnis, dass der positive prognostische Effekt von MSI-H auch dann erhalten bleibt, wenn die Patienten mit 5-FU behandelt werden (Widerspruch zu [14]) $[15,16]$.

\section{Fazit}

Ein Kolonkarzinom im Stadium II ist wahrscheinlich keine andere Erkrankung als ein Kolonkarzinom im Stadium III. Bei einer Überprüfung von 375 darmkrebsassoziierten Genen konnte nur bei 5 Genen ein signifikanter Unterschied bezüglich der Genexpression im Stadium II versus III festgestellt werden. MSI bleibt ein starker prognostischer Faktor im Stadium II; ob es auch ein prädiktiver Marker ist, ist aufgrund der widersprüchlichen Daten wieder offen. Vor diesem Hintergrund konnte sich die Expertenrunde zu keiner einheitlichen Empfehlung durchringen. Manche wollten die Testung aufrechterhalten, jedoch mit der Begründung, dann diejenigen Stadium-II-Patienten von der Therapie auszuschließen, die auch ohne Therapie eine gute Prognose haben. Mehrheitlich überwog jedoch die Empfehlung, MSI nicht mehr zu testen und die Therapieentscheidung unabhängig vom MSI-Status zu treffen, da die MSI-Testung derzeit keine evidenzbasierten praktischen Auswirkungen hat.
Der Nutzen von XELOX (Capecitabin/Oxaliplatin) blieb erhalten und nahm mit der Zeit weiter zu

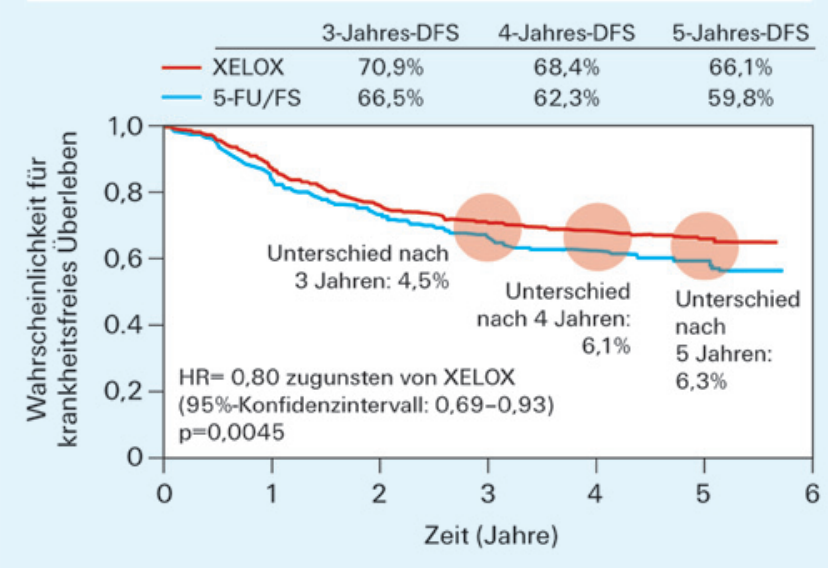

ITT Population

Abb. 2. NO16968 (XELOXA)-Studie: 5-Jahres-krankheitsfreies Überleben (Intention-to-Treat (ITT)-Analyse) (nach [30]).

\section{Stadium III - Standards und neue Daten}

Bei Patienten mit einem R0-resezierten Kolonkarzinom im Stadium III ist postoperativ eine Oxaliplatin-haltige Chemotherapie der Standard [1]. Diese klare Empfehlung der deutschen S3-Leitlinie basierte ursprünglich auf den Daten der Multicenter International Study of Oxaliplatin/5-Fluorouracil/ Leucovorin in the Adjuvant Treatment of Colon Cancer (MOSAIC)-Studie [3, 4], ergänzt durch die Ergebnisse der amerikanischen Studie National Surgical Adjuvant Breast and Bowel Project (NSABP) C-07 [17], denn in beiden Studien hatte die Kombination mit Oxaliplatin zu einem signifikant besseren krankheitsfreien Überleben (DFS) geführt als die alleinige 5-FU/FS-Gabe.

Seit dem letzten Jahr liegen jetzt die Ergebnisse einer weiteren Phase-III-Studie vor [18]. In dieser Studie, bekannt unter dem Namen NO16968 oder XELOXA (Xeloda plus Oxaliplation Adjuvant), hatten insgesamt 1886 Stadium-IIIPatienten postoperativ randomisiert entweder im experimentellen Arm das orale Fluoropyrimidin Capecitabin mit Oxaliplatin (XELOX) oder den früheren Standard, Bolus-5-FU/FS nach entweder dem Mayo-Clinic- oder dem Roswell-ParkSchema, erhalten. Die Kombinationstherapie führte zu einer signifikanten Verlängerung des DFS, dem primären Studienziel (Abb. 2). Der Vergleich mit den Ergebnissen aus MOSAIC und NSABP C-07 zeigt, dass alle 3 Kombinationsschemata eine in etwa vergleichbare Wirksamkeit, auch gegenüber der im jeweiligen Kontrollarm geprüften Monotherapie aufweisen.

Damit steht jetzt mit Capecitabin/Oxaliplatin (XELOX) ein weiteres Kombinationsregime für die adjuvante Therapie im UICC (Union internationale contre le cancer)-Stadium III 
zur Verfügung, das zudem den Vorteil bietet, dass die Patienten nicht mehr mit Pumpen oder Kathetern belasten werden müssen. Intravenöse 5-FU-basierte Protokolle sollten somit durch Capecitabin/Oxaliplatin ersetzt werden (positive Einschätzung der EMEA (European Medicines Agency) liegt vor, Zulassung wird bis Mitte April 2010 erteilt werden).

Auch die Verträglichkeit von XELOX unterscheidet sich deutlich von dem in der MOSAIC-Studie geprüften FOLFOX4-Regime (infusionales 5-FU/FS/Oxaliplatin). So traten unter XELOX erheblich weniger schwere Neutropenien $(8,8 \%$ vs. $41,1 \%)$, weniger febrile Neutropenien $(0,4 \%$ vs. $1,8 \%)$ und weniger schwere Stomatitiden ( $0,6 \%$ vs. $2,7 \%)$ auf, dafür aber deutlich mehr Diarrhoen (19,4\% vs. 10,8\%) und Hand-Fuß-Syndrome (5,4\% vs. 2,0\%) (Cross-Trial Comparison) [3, 19]. Das in der NSABP C-07-Studie geprüfte FLOX-Regime (Bolus-5-FU/FS/Oxaliplatin) wird aufgrund seiner schlechteren Verträglichkeit (38\% schwere Diarrhoen) und der erhöhten Mortalität nicht empfohlen.

Irinotecan-haltige Schemata sind in der Adjuvanz nicht von Nutzen, wie die Studien Cancer and Leukemia Group B (CALGB) 89803, PETACC-3 und Action to Control Cardiovascular Risk in Diabetes (ACCORD) gezeigt haben [16, 20, 21].

\section{Zielgerichtete Therapien in der adjuvanten Therapie}

Die auf dem letzten ASCO-Meeting vorgestellte Studie NSABP C-08 prüfte die zusätzliche Verabreichung von Bevacizumab zu einem modifizierten FOLFOX6-Regime [22]. Im Laufe des ersten Studienjahres war das Ergebnis signifikant zugunsten von Bevacizumab. Nach 3 Jahren zeigte sich jedoch bezüglich des primären Endpunktes (DFS) kein signifikanter Unterschied mehr, so dass die Studie insgesamt negativ ist. Die Ergebnisse einer weiteren Phase-III-Studie zum Einsatz des vaskulären endothelialen Wachstumsfaktor (VEGF)-Antikörpers Bevacizumab in der Adjuvanz (AVastin adjuvANT (AVANT)-Studie) stehen derzeit noch aus.

Der epidermale Wachstumsfaktorrezeptor (EGFR)-Antikörper Cetuximab wurde in einem vergleichbaren Setting ebenfalls in Kombination mit mFOLFOX6 geprüft, versus mFOLFOX6 allein (Studie North Central Cancer Treatment Group (NCCTG)-N0147, Eastern Cooperative Oncology Group (ECOG)-N0147). Die Ergebnisse sind noch nicht publiziert; einer Pressemeldung vom 13. Januar 2010 zufolge ist aber auch diese Studie negativ.

\section{Vorgehen bei älteren Patienten im Stadium III}

Frühere Studien haben gezeigt, dass über 70-jährige Patienten in vergleichbarer Weise von einer 5-FU-Monotherapie profitieren wie jüngere Patienten - und dass bei den über 70-Jährigen keine erhöhte Toxizität beobachtet wird [23]. Dies hatte zur Folge, dass die deutsche S3-Leitlinie 2008 ausdrücklich vermerkt hat, dass für die Durchführung einer adjuvanten Chemotherapie keine Altersbeschränkung existiert [1].

Die Frage, inwieweit ältere Patienten auch von einer Kombinationschemotherapie profitieren, wird in der genannten
Analyse [23] aber nicht beantwortet. Diese Frage war Gegenstand eines aktuellen Amendments zur S3-Leitlinie und wird derzeit wieder kontrovers diskutiert.

Ausgangspunkt der aktuellen Diskussion waren zum einen Sicherheitsbedenken, die im Rahmen der laufenden PETACC-8 Studie und der NSABP C-08-Studie aufgetreten waren. In der PETACC-8 Studie (adjuvant FOLFOX \pm Cetuximab) waren vermehrt therapieassoziierte Todesfälle bei über 70-Jährigen aufgetreten, und zwar auch bei den Patienten, die nur FOLFOX erhalten hatten, so dass die Studie für über 70-jährige Patienten geschlossen wurde. In der NSABP C-08-Studie sollen bei den Älteren vermehrt Grad-5-SAEs (serious adverse events) beobachtet worden sein [24].

Zum anderen wies eine Analyse der "ACCENT Collaborative Group», die auf dem letzten ASCO-Meeting vorgestellt wurde [25], auf eine geringere Effektivität von «neueren» adjuvanten Protokollen bei Älteren hin. In dieser Hypothesen generierenden Analyse sollte der Einfluss des Lebensalters auf die Wirksamkeit der «neueren Therapien» untersucht werden. Die Datenbasis waren dabei 6 aktuellere Phase-IIIStudien $[3,4,16,17,20,26-28]$, die alle «neuere Therapien» im Sinne von oralen Fluoropyrimidinen oder Oxaliplatin oder Irinotecan untersucht hatten. Insgesamt wurden 12669 Patienten analysiert, wobei der Anteil der über 70-Jährigen 17\% betrug. Das Gesamtergebnis zeigte einen signifikanten Nutzen im Hinblick auf das DFS (Hazard Ratio (HR) 0,85; Konfidenzintervall (CI) 0,80-0,91) und das Gesamtüberleben (HR 0,86; CI 0,79-0,92) für die unter 70-Jährigen, aber keinen Nutzen für die über 70-Jährigen (DFS: HR 1,11; CI 0,97-1,27; Gesamtüberleben: HR 1,14; CI 0,98-1,32).

Aufgrund des weiten Einschlusskriteriums «neuere Therapien» und der Heterogenität hinsichtlich der Substanzen und des Studiendesigns (teils mit Nichtunterlegenheits-, teils mit Überlegensheitshypothesen) interessieren vor allem die beiden Studien mit der Oxaliplatin-haltigen Kombinationstherapie, MOSAIC und NSABP C-07 [3, 4, 17]. Auch in diesen Studien konnte ein signifikanter Nutzen hinsichtlich des krankheitsfreien und des Gesamtüberlebens bei Patienten im Stadium III nur für die unter 70-Jährigen $(\mathrm{n}=3977)$, nicht aber für die über 70-Jährigen $(\mathrm{n}=703)$ dokumentiert werden (DFS: HR 0,77 (CI: 0,68-0,86) bzw. 1,04 (CI: 0,80-1,35); Gesamtüberleben: HR 0,81 (CI: 0,71-0,93) bzw. 1,19 (CI: 0,901,57); Test auf Interaktion: $\mathrm{p}=0,016$ bzw. 0,037).

Dies führte Ende des Jahres 2009 zu der Konsequenz, die S3-Leitlinie per Konsensus dahingehend zu modifizieren, dass nur noch für die unter 70-jährigen Patienten eine klare Empfehlung für Oxaliplatin-haltige Protokolle formuliert wurde. Für Patienten über 70 Jahre wurde eine zurückhaltende Indikationsstellung für die Oxaliplatin-Kombinationstherapie angeraten. Unstrittig ist bei Patienten über 70 Jahre aber eine Fluoropyrimidin-Monotherapie empfohlen worden [29].

Aktuell (auf dem ASCO Gastrointestinal Cancers Symposium im Januar 2010) wurde eine Subgruppenanalyse der oben beschriebenen XELOXA-Studie [18] vorgestellt, die 
Tab. 1. NO16968 (XELOXA)-Studie: Subgruppenanalyse: Krankheitsfreies Überleben in Abhängigkeit vom Alter

\begin{tabular}{llll}
\hline & \multicolumn{2}{l}{ 3-Jahres-DFS } & \multirow{2}{*}{ Hazard Ratio (95\%-CI) } \\
\cline { 2 - 3 } & XELOX & 5-FU/FS & \\
\hline$<65$ vs. $>65$ Jahre & $72 \%$ & $69 \%$ & $0,80(0,65-0,98)$ \\
$<65$ Jahre, $\mathrm{n}=1142$ & $68 \%$ & $62 \%$ & $0,81(064-1,03)$ \\
$\begin{array}{l}\geq 65 \text { Jahre, } \mathrm{n}=744 \\
<70 \text { vs. }>70 \text { Jahre }\end{array}$ & & & \\
$<70$ Jahre, $\mathrm{n}=1477$ & $72 \%$ & $69 \%$ & $0,79(0,66-0,94)$ \\
$\geq 70$ Jahre, $\mathrm{n}=409$ & $66 \%$ & $60 \%$ & $0,87(0,63-1,18)$ \\
\hline
\end{tabular}

Nach [30].

DFS = Krankheitsfreies Überleben, CI = Konfidenzintervall. den Nutzen der Kombination Capecitabin/Oxaliplatin unabhängig vom Alter - und explizit auch bei den über 70-jährigen Patienten - hinsichtlich der Verbesserung des DFS zeigt [30] (Tab. 1).

Dieses Ergebnis stellt die Effektivitätsaussage der ACCENT-Analyse erneut zur Diskussion. Die Expertenrunde empfahl deshalb, die Entscheidung, ob man einen über 70-jährigen Patienten nur mit einem (oralen) Fluoropyrimidin oder in Kombination mit Oxaliplatin behandelt, vor allem am biologischen Alter sowie den Komorbiditäten des Patienten festzumachen; wenn ein über 70-jähriger Patient die Eingangskriterien des XELOXA-Protokolls erfüllt, wäre der Patient im Prinzip auch für XELOX geeignet.

Liegt eine Kontraindikation gegen Oxaliplatin vor, ist ohnehin eine Monotherapie mit Fluoropyrimidinen indiziert. Dabei werden orale Fluoropyrimidine den infusionalen Schemata vorgezogen. Der Einsatz von 5-FU-Bolus-Regimen gilt als obsolet [1]. Diese Empfehlung der S3-Leitlinie basiert im Wesentlichen auf den Ergebnissen der Xeloda in Adjuvant Colon Cancer Therapy (X-ACT)-Studie, die zeigen konnte, dass eine Capecitabin-Monotherapie hinsichtlich des DFS (primärer Endpunkt) einem 5-FU-Bolus-Schema nicht unterlegen ist $[27,28]$.

Fazit

Mit Capecitabin/Oxaliplatin (XELOX) steht jetzt ein weiteres wirksames Kombinationsregime für die adjuvante Therapie im Stadium III zur Verfügung. Die Daten der ACCENT-Analyse werden durch eine aktuelle Subgruppenanalyse der XELOXA-Studie relativiert. Die Expertenrunde empfahl deshalb, die Entscheidung, ob man einen über 70-jährigen Patienten nur mit einem (oralen) Fluoropyrimidin oder in Kombination mit Oxaliplatin behandelt, mehr denn je am biologischen Alter und den Komorbiditäten des Patienten festzumachen.

\section{Disclosure Statement}

The authors were speakers at the meeting and contributors to the publication 'X. Interdisziplinärer Expertenworkshop "Gastrointestinale Tumore", Schlossgut Oberambach, Münsing', sponsored by Roche Pharma AG.

\section{Literatur}

1 Schmiegel W, et al.: S3-Leitlinie «Kolorektales Karzinom» - Aktualisierung 2008. Z Gastroenterol 2008;46:799-840.

2 QUASAR Collaborative Group: Adjuvant chemotherapy versus observation in patients with colorectal cancer: a randomised study. Lancet 2007;370: 2020-2029.

3 Andre T, et al.; Multicenter International Study of Oxaliplatin/5-Fluorouracil/Leucovorin in the Adjuvant Treatment of Colon Cancer (MOSAIC) Investigators: Oxaliplatin, fluorouracil, and leucovorin as adjuvant treatment for colon cancer. N Engl J Med 2004;350:2343-2351.

4 André $\mathrm{T}$, et al.: Improved overall survival with oxaliplatin, fluorouracil, and leucovorin as adjuvant treatment in stage II or III colon cancer in the MOSAIC trial. J Clin Oncol. 2009;27:3109-3116.

5 Roth AD, et al.: Stage-specific prognostic value of molecular markers in colon cancer: Results of the translational study on the PETACC 3-EORTC 40993-SAKK 60-00 trial. J Clin Oncol 2009;27 (suppl):15s, abstr 4002 .
6 Roth AD, et al.: Prognostic role of KRAS and BRAF in stage II and III resected colon cancer: results of the translational study on the PETACC-3, EORTC 40993, SAKK 60-00 trial. J Clin Oncol 2010;28:466-474.

7 Popat S, et al.: Systematic review of microsatellite instability and colorectal cancer prognosis. J Clin Oncol 2005;23:609-618.

8 O'Connell MJ, et al.: Survival following recurrence in stage II and III colon cancer: findings from the ACCENT data set. J Clin Oncol 2008;26:2336-2341.

9 O'Connell MJ, Lavery IC, Gray RG, et al.: Comparison of molecular and pathologic features of stage II and stage III colon cancer in four large studies conducted for development of the 12-gene colon cancer recurrence score. Orlando, ASCO Gastrointestinal Cancers Symposium 2010, abstr 280.

10 Kerr D, et al.: A quantitative multigene RT-PCR assay for prediction of recurrence in stage II colon cancer: Selection of the genes in four large studies and results of the independent, prospectively designed QUASAR validation study. J Clin Oncol 2009;27(suppl):15s, abstr 4000
11 Salazar R, et al.: Development and validation of a robust high-throughput gene expression test (ColoPrint) for risk stratification of colon cancer patients. Orlando, ASCO Gastrointestinal Cancers Symposium 2010, abstr 295.

12 Ribic CM, et al.: Tumor microsatellite-instability status as a predictor of benefit from fluorouracilbased adjuvant chemotherapy for colon cancer. N Engl J Med. 2003;349:247-257.

13 Jover R, et al.: The efficacy of adjuvant chemotherapy with 5-fluorouracil in colorectal cancer depends on the mismatch repair status. Eur J Cancer 2009;45:365-373.

14 Sargent DJ, et al.: Confirmation of deficient mismatch repair (dMMR) as a predictive marker for lack of benefit from 5-FU based chemotherapy in stage II and III colon cancer (CC): A pooled molecular reanalysis of randomized chemotherapy trials. Orlando, ASCO Gastrointestinal Cancers Symposium 2008, abstr 4008 .

Reinacher-Schick/Arnold/Trarbach/Ridwelski/ Bruch/Kirchner/Kubicka/Schmoll 
15 Tejpar S, et al.: Microsatellite instability (MSI) in stage II and III colon cancer treated with 5FU-LV or 5FU-LV and irinotecan (PETACC 3-EORTC 40993-SAKK 60/00 trial). J Clin Oncol 2009;27 (suppl):15s, abstr 4001.

16 Van Cutsem E, et al.: Randomized phase III trial comparing biweekly infusional fluorouracil/leucovorin alone or with irinotecan in the adjuvant treatment of stage III colon cancer: PETACC-3. J Clin Oncol 2009;27:3117-3125.

17 Kuebler JP, et al.: Oxaliplatin combined with weekly bolus fluorouracil and leucovorin as surgical adjuvant chemotherapy for stage II and III colon cancer: results from NSABP C-07. J Clin Oncol 2007;25:2198-2204.

18 Haller DG, et al.: NO16968: XELOXA. Adjuvant treatment with capecitabine and oxaliplatin (XELOX) in stage III colon cancer. Berlin, ESMO/ ECCO Presidential Session III, 2009.

19 Schmoll HJ, et al.: Phase III trial of capecitabine plus oxaliplatin as adjuvant therapy for stage III colon cancer: a planned safety analysis in 1,864 patients. J Clin Oncol 2007;25:102-109.

20 Saltz LB, et al.: Irinotecan fluorouracil plus leucovorin is not superior to fluorouracil plus leucovorin alone as adjuvant treatment for stage III colon cancer: results of CALGB 89803. J Clin Oncol 2007; 25:3456-3461.
21 Ychou M, et al:: A phase III randomized trial of LV5FU2+CPT-11 vs. LV5FU2 alone in adjuvant high risk colon cancer (FNCLCC Accord02/ FFCD9802). Orlando, ASCO Gastrointestinal Cancers Symposium 2005, abstr 3502.

22 Wolmark N, et al.: A phase III trial comparing mFOLFOX6 to mFOLFOX6 plus bevacizumab in stage II or III carcinoma of the colon: Results of NSABP Protocol C-08. J Clin Oncol 2009;27 (suppl):18s, abstr LBA4.

23 Sargent DJ, et al.: A pooled analysis of adjuvant chemotherapy for resected colon cancer in elderly patients. N Engl J Med 2001;345:1091-1097.

24 Allegra CJ, et al.: Initial safety report of NSABP C-08: A randomized phase III study of modified FOLFOX6 with or without bevacizumab for the adjuvant treatment of patients with stage II or III colon cancer. J Clin Oncol 2009;27:3385-3390.

25 Jackson McCleary NA, et al.: Impact of older age on the efficacy of newer adjuvant therapies in $>12500$ patients (pts) with stage II/III colon cancer: Findings from the ACCENT database. J Clin Oncol 2009;27(suppl):15s, abstr 4010.

26 Lembersky BC, et al.: Oral uracil and tegafur plus leucovorin compared with intravenous fluorouracil and leucovorin in stage II and III carcinoma of the colon: results from National Surgical Adjuvant Breast and Bowel Project Protocol C-06. J Clin Oncol 2006;24:2059-2064.
27 Twelves C, et al.: Capecitabine as adjuvant treatment for stage III colon cancer. N Engl J Med 2005;352:2696-2704.

28 Twelves $\mathrm{C}$, et al.: Capecitabine versus 5-FU/LV in stage III colon cancer: Updated 5-year efficacy data from X-ACT trial and preliminary analysis of relationship between hand-foot syndrome (HFS) and efficacy. Orlando, ASCO Gastrointestinal Cancers Symposium 2008, abstr 274

29 Schmiegel W, et al.: Colorectal carcinoma: the management of polyps, (neo)adjuvant therapy, and the treatment of metastases. Dtsch Arztebl Int 2009;106:843-848.

30 Haller DG, et al.: Efficacy findings from a randomized phase III trial of capecitabine plus oxaliplatin versus bolus 5-FU/LV for stage III colon cancer (NO16968): No impact of age on disease-free survival (DFS). Orlando, ASCO Gastrointestinal Cancers Symposium 2010, abstr 284.

31 Reinacher-Schick A, et al.: Chemotherapy of colorectal cancers [in German]. Internist 2009;50:12391252 . 